\title{
Organic Synthesis without Stoichiometric Reagents: A Guiding Principle for Reaction Development
}

\author{
Jeffrey W. Bode*
}

\begin{abstract}
A common theme of our research program is the development of new organic transformations that operate under catalytic conditions or as ligation reactions that do not require the addition of any reagents or other additives. Our catalysis program features the transient generation of reactive species from alpha-functionalized aldehydes via intramolecular redox reactions using $\mathrm{N}$-heterocyclic carbenes as multifunctional catalysts. This approach makes possible the catalytic generation of enolates, homoenolates, and activated carboxylates and their application to diastereo- and enantioselective transformation. Intermolecular redox couplings are key to a general, highly chemoselective amide-forming ligation reaction and its use for oligopeptide synthesis. The concepts behind these transformation and examples of their use as well as current and future directions of our research program are presented.
\end{abstract}

Keywords: Catalysis · Ligation · Reactions · Stereochemistry $\cdot$ Synthesis

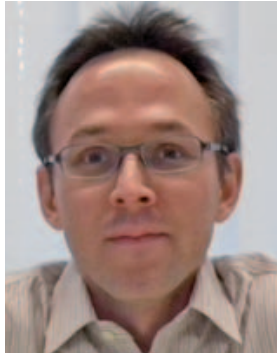

Jeffrey Bode is Professor of Synthetic Organic Chemistry at ETH Zürich. His research group works on the development of new reactions and their application to the synthesis of small molecules and peptides, shapeshifting organic molecules, and molecular diagnostics. Following graduate studies at the California Institute of Technology and ETH Zürich he was a JSPS postdoctoral fellow at the Tokyo Institute of Technology. In 2003 he became Assistant Professor of Chemistry and Biochemistry at the University of California, Santa Barbara and moved as Associate Professor to the University of Pennsylvania in 2007. In 2010 he returned to Zürich to take up his present position. Jeffrey's research and teaching have been recognized by numerous awards, most recently the E. J. Corey Award for Outstanding Contributions to Organic Synthesis by a Young Investigator from the American Chemical Society.

${ }^{\star}$ Correspondence: Prof. J. W. Bode ETH Zürich

Laboratorium für Organische Chemie

Wolfgang Pauli Strasse 10

$\mathrm{CH}-8093$ Zürich

Tel. +41446332103

E-mail: bode@org.chem.ethz.ch

\section{Introduction}

It was with great pleasure - along with some trepidation - that I had the opportunity to return to Switzerland in 2010, nearly ten years after finishing my Dok. Nat. Science at ETH Zürich in 2001. My first introduction to Switzerland and ETH came in 1998 when I found myself, then as a second year graduate student, suddenly transported to Zurich as part of the research group of Prof. Erick Carreira. Our move to ETH was an exciting opportunity to learn about the intensity, creativity, hospitality, and dynamics of the Swiss chemical community. These memories were not lost during postdoctoral studies in Japan with Prof. Keisuke Suzuki (who also has a strong connection to ETH and Switzerland) and the beginning of my independent academic career in the United States. It was therefore both an incredible privilege and in many ways a dream come true to receive a call to return to ETH and join my many friends and colleagues in Switzerland and Europe. The scientific opportunities here are limitless and the many connections to other researchers in both academics and industries gives us the impetus to not only look far into the future in formulating our long term scientific goals but also to look closely at unsolved contemporary problems in need of immediate solutions.

The overarching goal of our research program is the identification of new reactions, new concepts, and new challenges for the synthesis of complex molecules and materials. A motivation for much of our work is the growing recognition that the next generation of medicines and mate- rials will fall into a range of size and complexity that is currently not well served by either synthetic chemistry or bioengineering techniques. Our projects are a balance between developing the methods and techniques that will play a role in pushing the boundaries of synthetic chemistry and providing new reactions and catalyst that impact contemporary issues of synthesis, selectivity, and sustainability.

In each of these pursuits, we have endeavored to develop reactions and processes that do not require stoichiometric quantities of reagents - or even reagents at all. To achieve this we have been advancing redox neutral reactions in which there is no net change in the oxidation state of the two reactants. This has already proven to be a remarkably powerful design consideration for both novel catalytic and enantioselective reactions using $\mathrm{N}$-heterocyclic carbenes and the development of a chemoselective amide ligation. We are the first to point out that our strategies requires the use of specialized functional groups or reactants primed for specific and selective coupling reactions. Their preparation requires reagents - and relies on remarkable advances in synthetic organic chemistry pioneered by many research groups. But we believe that the strategy of segregating the preparation of advanced building blocks and general methods for their coupling together is powerful concept for accessing larger and more complex organic structures, with less time required for synthesis and purification. This approach is inspired by widespread strategies for oligopeptide synthesis and transition-metal medi- 
ated cross coupling. Our emphasis is on the development of a new generation of coupling reactions that obviate the need for reagents, provide novel reaction modalities, and ideally operate in the presence of the unprotected functional groups typically found in contemporary synthetic targets.

The intention of this account is to give a sense of the problems in synthetic methodology that we are currently interrogating and the mechanistic principles that guide our discovery. As part of our move to ETH Zürich, we are currently applying these reactions and concepts in more ambitious ways with the goal of addressing what we feel are some of the most pressing and challenging problems in organic synthesis including the preparation of biologically derived molecules, chemoselective functionalization, the synthesis of cyclic peptides and other macrocycles, controlled oligomerization and polymerization, and the development of new molecular probes for the detection, diagnosis and treatment of human disease. The work described should be considered a preamble to future work towards these general goals rather than self-contained research programs.

\section{Catalytic Generation of Reactive Intermediates from $\alpha$-Functionalized Aldehydes}

A common practice in synthetic organic chemistry is the generation of highly reactive species as the key intermediates for constructing $\mathrm{C}-\mathrm{C}, \mathrm{C}-\mathrm{N}$, and $\mathrm{C}-\mathrm{O}$ bonds. For example, the formation of Grignard reagents from organohalides is a reliable and widely employed approach to $\mathrm{C}-\mathrm{C}$ bond formation. The limitation of such methods is the relatively harsh reaction conditions that demand thorough protection of most functional groups and the exclusion of water, as well as the need for superstoichiometric amounts of reagents that result in reaction byproducts. It is therefore no surprise that synthetic chemists have made major strides towards the catalytic generation of reactive intermediates to effect such reactions under operationally friendly conditions without the need for stoichiometric reagents. The catalytic generation of reaction species also provides a platform for the development of catalytic enantioselective methods. Notable examples including direct aldol-type reactions of aldehydes and ketones under proline catalysis, ${ }^{[1]}$ the addition of terminal acetylenes to aldehydes and imines, ${ }^{[2]}$ and the reductive generation of nucleophiles from alkenes and alkynes with transition metals. ${ }^{[3]}$

In our own research we have sought to develop methods for the catalytic generation of reactive intermediates for which

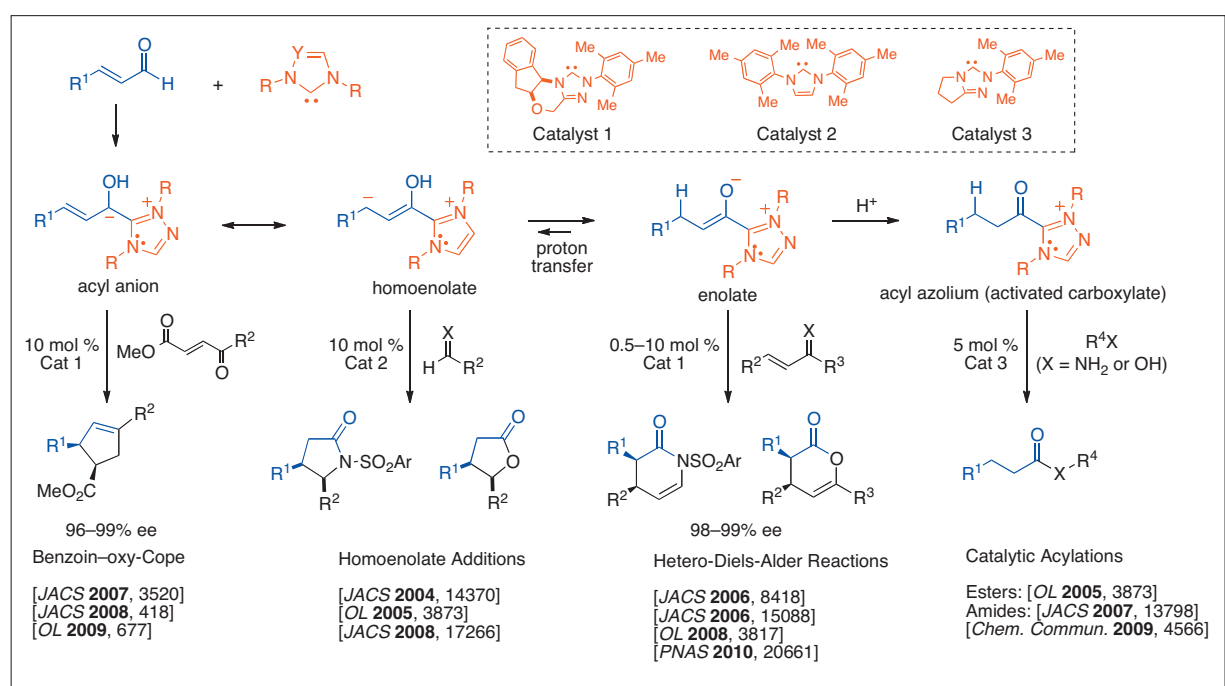

Scheme 1. NHC-catalyzed generation of reactive species developed in the Bode Group.

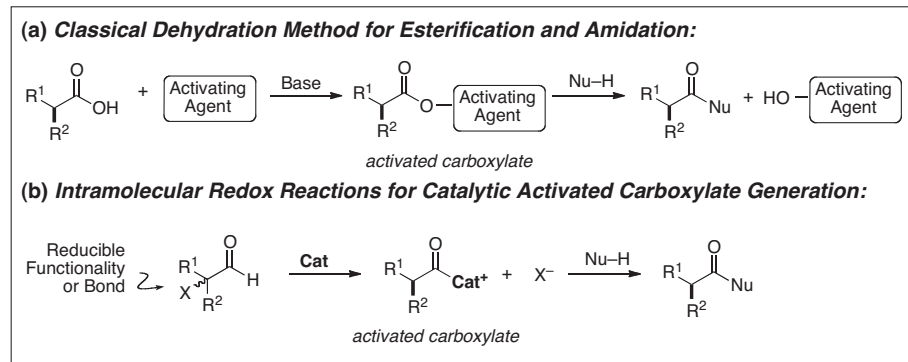

Scheme 2. Methods for the synthesis of activated carboxylates.

there are few methods for their substoichiometric preparation. Our initial target of catalytically generating activated carboxylates has now expanded to include the generation of homoenolates and ester enolate equivalents, all from the same class of starting materials. To achieve this, we have pioneered $\mathrm{N}$-heterocyclic carbene catalyzed generation of reactive intermediates by internal redox reactions of $\alpha$-functionalized aldehydes. As our research has evolved, we have successfully developed reaction conditions and catalysts for the selective generation of each of these reactive intermediates from enals and their surrogates. Furthermore, many of these reactive species can be generated from other $\alpha$-functionalized aldehydes including $\alpha$-halo aldehydes, $\alpha, \beta$-epoxyaldehydes, and formyl cyclopropanes.

\subsection{Catalytic Generation of Activated Carboxylates}

The typical synthesis of carboxylic esters, amides, and thioesters prescribes super-stoichiometric amounts of expensive coupling reagents, bases, and additives to effect the union of a carboxylic acid and a nucleophile (Scheme 1). Such reactions routinely produce several equivalents or more of byproducts, necessitating complicated workups or other strategies, such as solid supports, to isolate the desired products. Furthermore, despite the ubiquity of carboxylic acid derivatives containing neighboring stereocenters, i.e. amino acid residues, there are few catalytic methodologies for the direct synthesis of carboxylic acid derivatives from constituent fragments with simultaneous introduction or control of stereochemical complexity. ${ }^{[4]}$ We found this surprising. Most carboxylic acid derivates are prepared via the transient intermediacy of a key species, an activated carboxylate, ${ }^{[5]}$ which renders the carboxylic acid amenable to nucleophilic attack. Given that these species are rarely isolated, a methodology for the catalytic generation of this key activated carboxylate should be synthetically tenable. ${ }^{[6]}$

With these goals in mind, we initiated a program aimed at the catalytic generation of activated carboxylates via catalyst induced intramolecular redox reactions (Scheme 2b). This methodology is predicated on the use of aldehydes or aldehyde equivalents poised for oxidation by reduction of an adjacent functionality, using the reactivity principles inherent to the benzoin reaction and the chemistry of azolium salts. Nucleophilic addition to the resulting acyl azolium results in a carboxylic acid derivative and regenerates the active catalyst rather than production of a reaction byproduct (Scheme 3). Our studies, and those of other groups, have now established that azolium-catalyzed redox esterification reactions are possible for most aldehydes bearing an $\alpha$-leaving group or unsaturatation. 


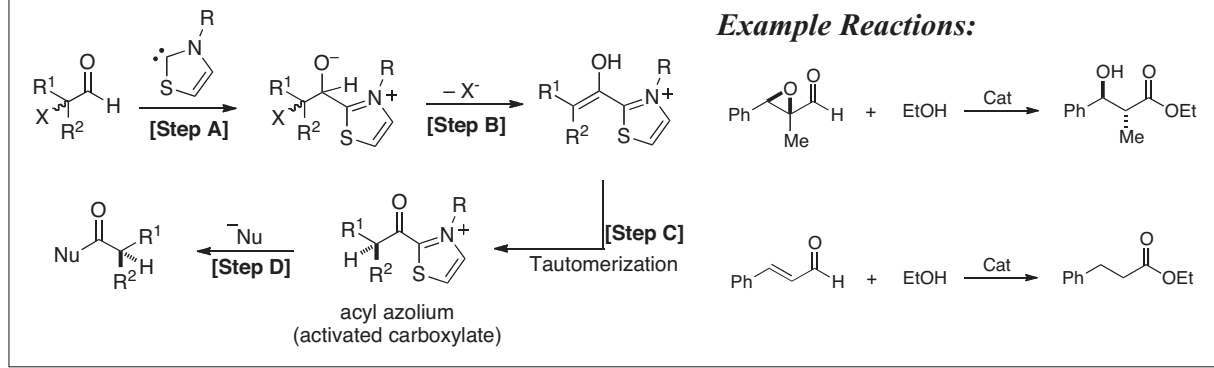

Scheme 3. Catalytic generation of activated carboxylates from $\alpha$-functionalized aldehydes.

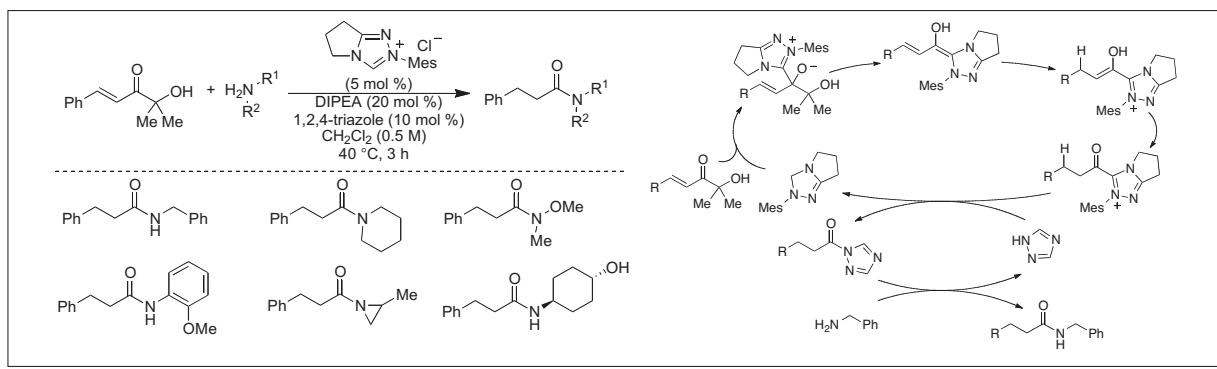

Scheme 4. Catalytic amide bond formation.

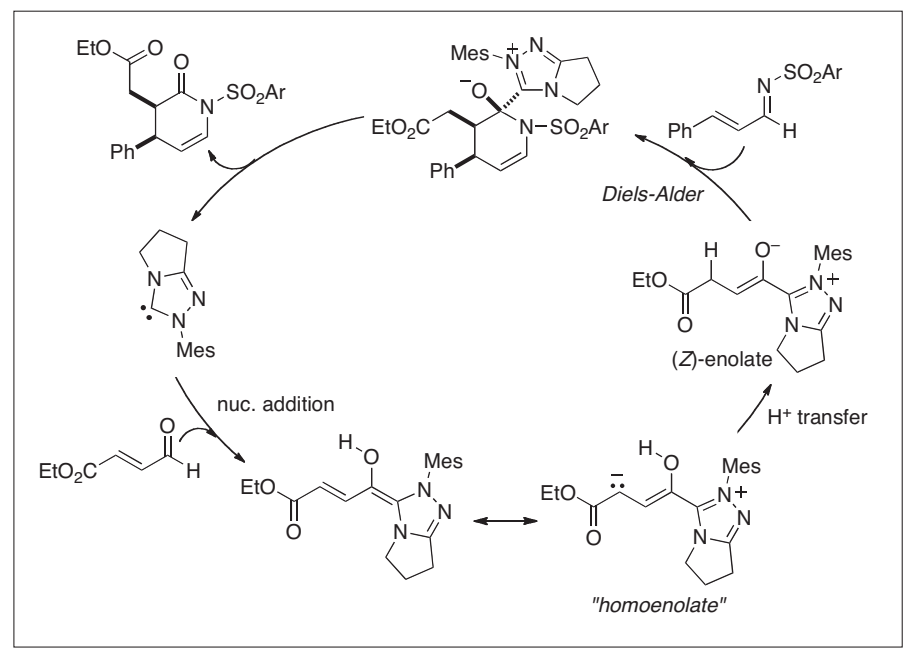

cat. A
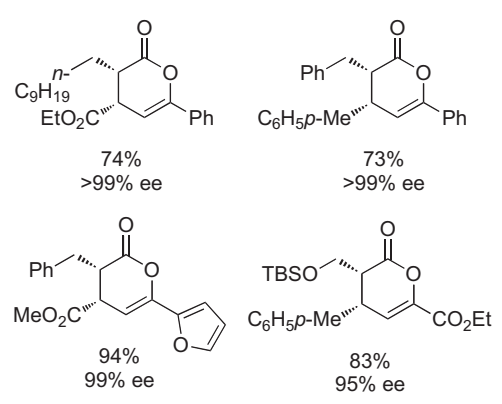

Fig. 2. Products prepared by NHC-catalyzed hetero-Diels Alder reactions.

A curious feature of acyl azolium species generated under these reaction conditions is their unusual chemoselectivity. Unlike almost all other activated carbox-
Fig. 1.
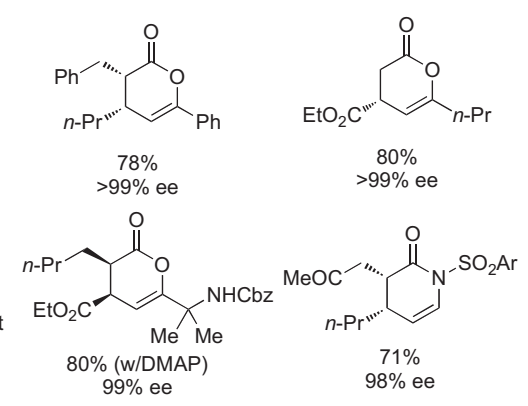

Scheme 5. NHCcatalyzed Diels-Alder reactions.

ylates, which are typically generated with stoichiometric reagents, acyl azolium show a strong preference for acylation of alcohols in preference to amines. ${ }^{[7]}$ This makes possible, for example, the chemoselective acylation of alcohols in the presence of amines. ${ }^{[8]}$ This unusual chemoselectivity has its uses and is a subject of continued study in our research group. Alternatively, the NHC-catalyzed generation of activated carboxylates can be used for amide formation by the inclusion of suitable cocatalysts such as imidazole, triazole, or HOAt (Scheme 4). ${ }^{[9]}$ As a further improvement, hydroxyenones can be used as surrogates for aldehydes.[10] This both simplifies the preparation of the starting materials and avoids the formation of imines during the reaction. The overall process is an example of two small molecules working in tandem to accomplish a reagentless transformation that is impossible for a single catalyst to accomplish alone.

\subsection{Catalytic Generation of Ester Enolate Equivalents}

The addition of carbanions to electrophiles is perhaps the most common method for the construction of carbon-carbon bonds. Few processes have been more studied and refined than the aldol reaction and its relatives. An era dominated by strategies for diastereo- and enantioselective carbonyl additions led to intense research on direct methods that did not require chiral auxiliaries or the generation of silylketene acetals. These efforts converged on the identification of Lewis acid catalysts for the direct, enantioselective addition of activated ketones to aldehydes and imines ${ }^{[11]}$ and proline-catalyzed enantioselective additions of aldehydes to a variety of electrophiles. ${ }^{[12]}$

We recognized that the NHC-catalyzed generation of activated carboxylates from $\alpha$-functionalized aldehydes must pass through an enol or enolate intermediate (Scheme 5). Initial attempts to generate and trap this intermediate using thiazolium or simple triazolium catalysts proved unsuccessful, which eventually led to our development of N-mesityl substituted triazolium salts and its chiral variants as effective catalysts (Fig. 1).

These NHC precursors are outstanding catalysts for the generation of chiral ester enolate equivalents from $\alpha$-functionalized aldehydes. This makes possible, for example, inverse demand Diels-Alder reactions with unsaturated imines and ketones in excellent yield and outstanding enantioselectivity. ${ }^{[13]}$ We are particularly pleased that numerous other groups have adopted these catalysts and strategy for the generation of ester enolate equivalents for a variety of new reactions including a variety of formal cycloadditions ${ }^{[14]}$ and catalytic enantioselective Mannich reactions (Fig. 2). ${ }^{[15]}$ 


\subsection{Catalytic Generation of Homoenolate Equivalents}

The conjugated Breslow intermediate that arises from the interaction of an $\mathrm{N}$-heterocyclic carbene catalysts and an $\alpha, \beta$-unsaturated aldehyde can be considered as a carbon nucleophile at either of the acyl position, as in the classical Benzoin or Stetter reactions, or at the carbon $\beta$-to the aldehyde (Scheme 6). This is formally a reversal of the usual reactivity of $\alpha, \beta$-unsaturated aldehydes and this species can react as either an acyl anion or homoenolate equivalent. In 2004, our group and that of Glorius reported the use of Nmesityl substituted imidazolium catalysts for the generation of such homoenolate equivalents for the synthesis of $\gamma$-lactones (Scheme 7).[16] These initial publications led to an explosion of new synthetic methods based on this concept, with excellent work coming from not only our own group but many others as well.[17]

The lists of reactions that proceed from this activation mode are now too numerous to list comprehensively. Instead we give a brief survey of reactions developed in our group over the past few years and refer readers to several excellent reviews that provide an overview of this rapidly changing area. ${ }^{[18]}$ The catalytic generation of homoenolates can be extended to annulation reactions with a variety of other nucleophiles including the synthesis of $\gamma$-lactams from enals and imines (Scheme 8). ${ }^{[19]}$ The use of other nitrogen electrophiles leads to other lactam products. ${ }^{[20]}$

Several groups, including our own, have attempted to trap catalytically generated homoenolates by conjugate acceptors. With electrophilic enones, this is possible although the products observed arise via a rather complicated, but remarkably clean, reaction cascade that delivers cyclopentenes or bicyclic cyclopentane-lactones (Scheme 9).[21,22]

\subsection{Enantioselective Coates- Claisen Rearrangements}

Early in the course of our investigations into the catalytically generated activated carboxylates and homoenolates from enals we recognized the possibility of using N-heterocyclic carbene catalysis to form yet another synthetically valuable, catalytically generated intermediate in the form of $\alpha, \beta$-unsaturated ester equivalents that would be expected to undergo conjugated addition reactions (Scheme 10). In theory, a chiral NHC-catalyst could control the absolute stereochemical outcome of the addition, leading to a 'transient chiral auxiliary' approach to conjugate addition. Using our redox neutral approach, these species would arise from the combination of an N-heterocyclic carbene catalysts and

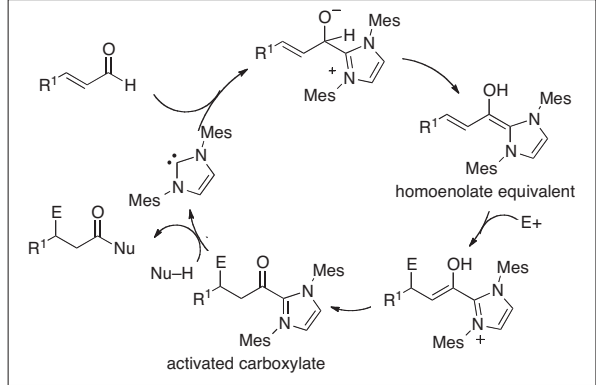

Scheme 6. Catalytic cycle for homoenolate generation.

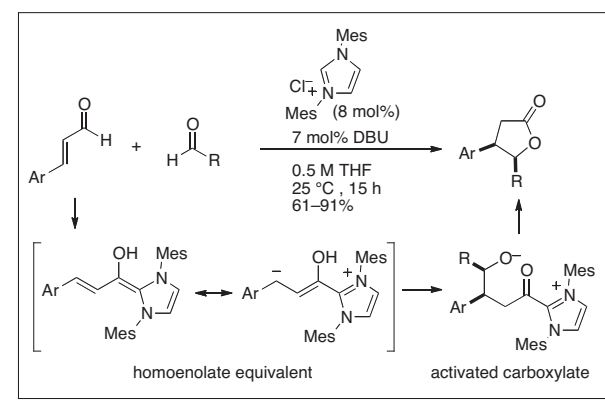

Scheme 7. Synthesis of $\gamma$-lactones by NHCcatalyzed annulations.

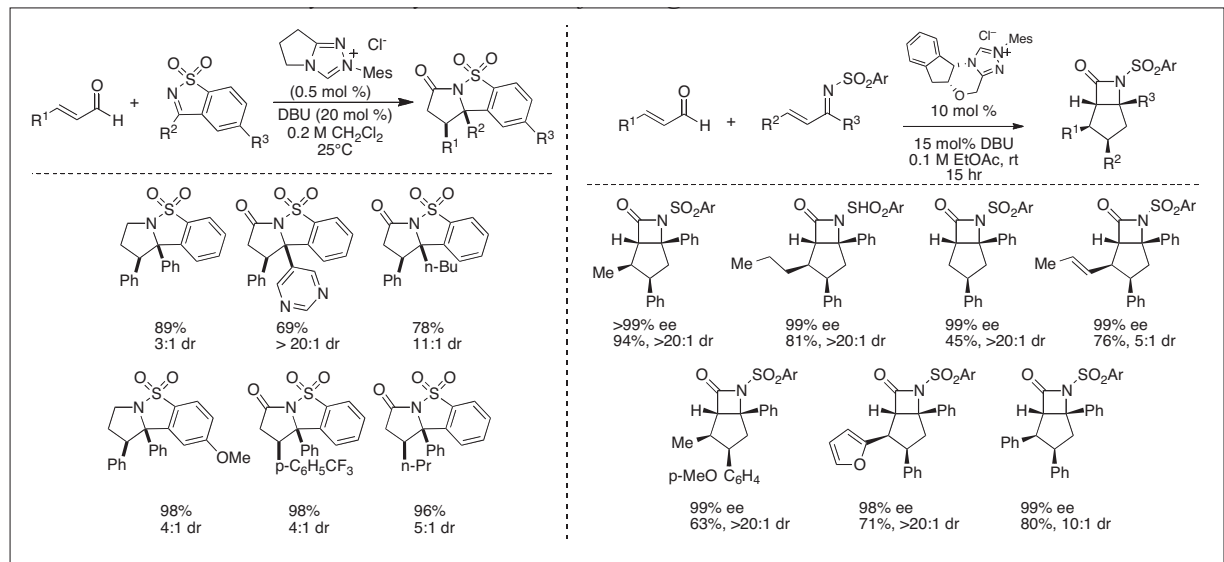

Scheme 8. NHC-catalyzed bicyclic-lactam forming annulations.

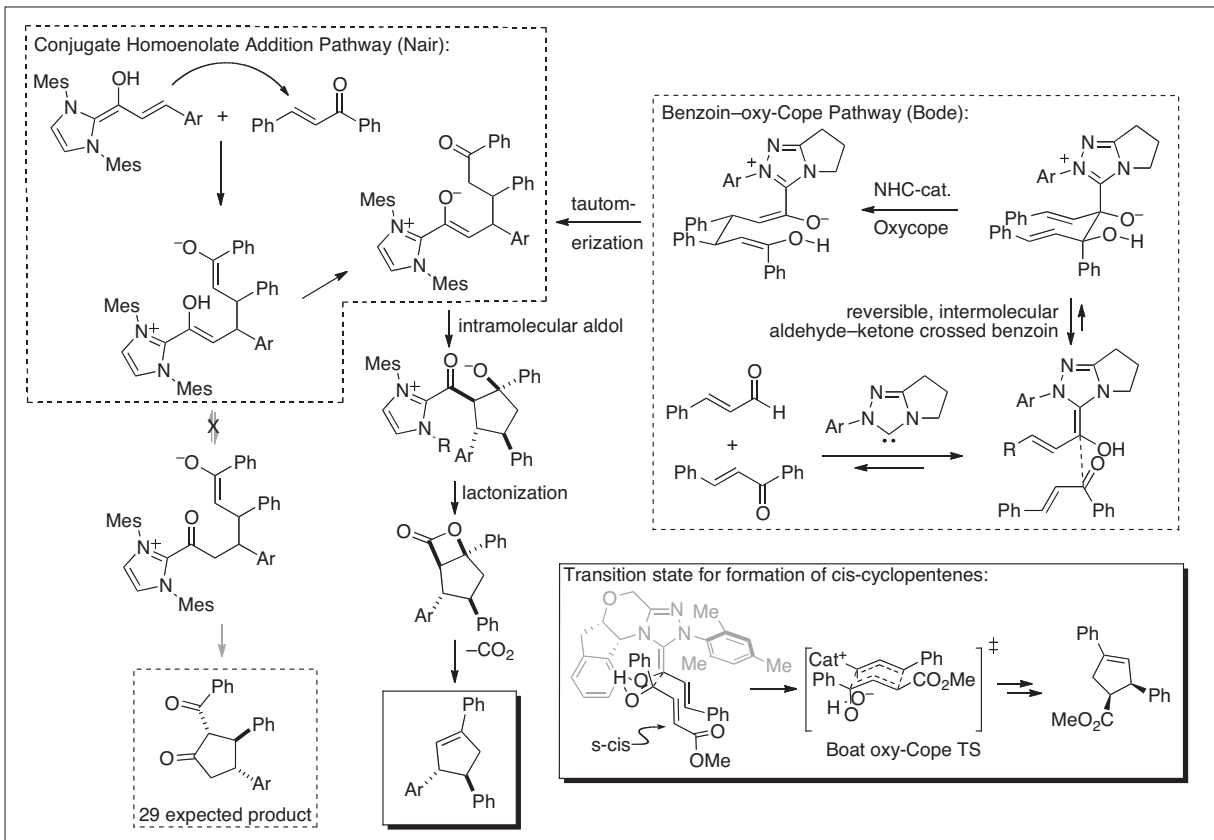

Scheme 9. Cyclopentene formation via NHC-catalyzed 1,4-additions of enals.

an ynal and such a process for the formation of $\alpha, \beta$-unsaturated esters was reported by Zeitler in 2005. Despite the ease of their generation under catalytic condition, all of our initial attempts to trap them with good nucleophiles proved fruitless and in most cases 1,2-adducts, rather than the desired 1,4-addition products, were formed instead.

The sole exception to this was the use of ketone-derived nucleophiles including
1,2- and 1,3-ketoesters. For example, the combination of ethyl pyruvate and an ynal in the presence of our N-mesityl substituted triazolium precatalysts leads to the formation of dihydropyranones products in good yield and outstanding enantioselectivity. Extensive mechanistic and kinetic investigations of this reaction, including the full characterization of the catalytically generated $\alpha, \beta$-unsaturated acyl azo- 


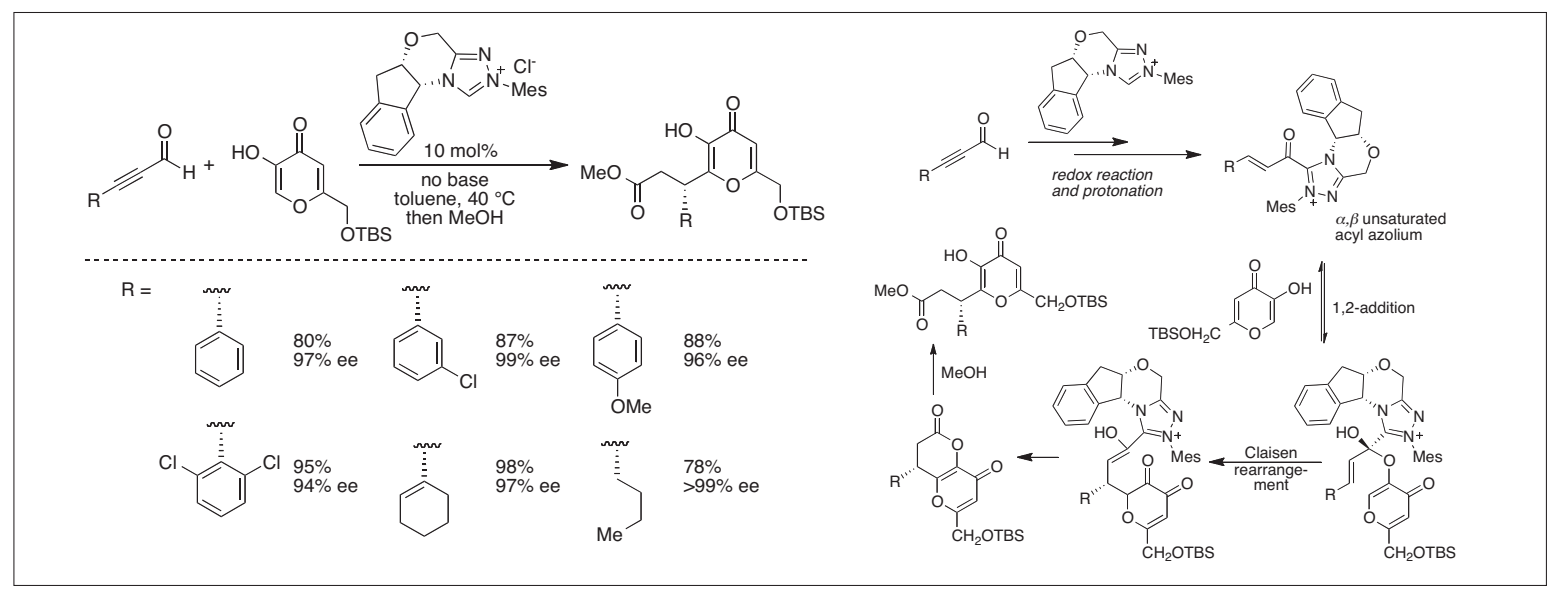

Scheme 10.

$\mathrm{NHC}$-catalyzed

enantioselective

Coates-Claisen

rearrangements.

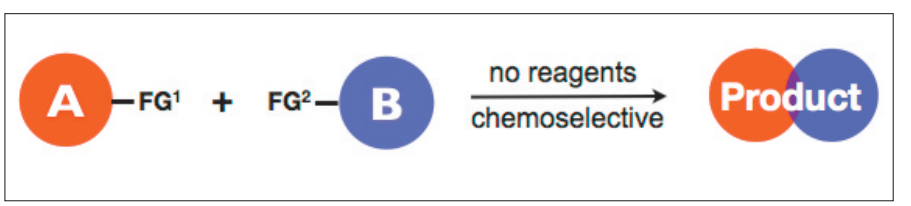

Scheme 11. Generic depiction of a ligation reaction.

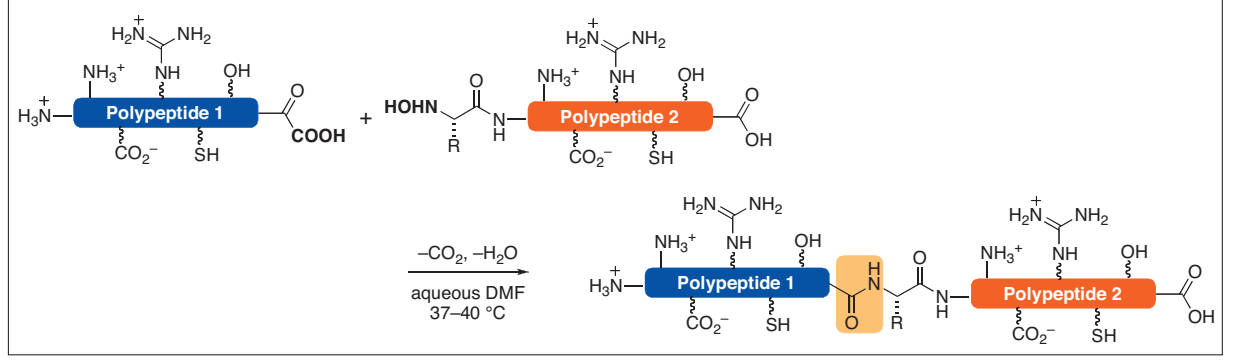

Scheme 12. Ketoacid-hydroxylamine amide ligations of unprotected biomolecules fragments.

lium intermediate revealed the annulation reaction is best characterized as an NHCcatalyzed variation of the Coates-Claisen reaction of $\alpha, \beta$-unsaturated acetals and enols. ${ }^{[23]}$ This led to our application of this reaction to Claisen rearrangements of kojic acid derivatives, which have demonstrated synthetic utility but which have resisted enantioselective synthesis using metalbased Claisen rearrangements. ${ }^{24]}$

\section{New Ligation Reactions for the Synthesis of Biomolecules and Biomaterials}

Our methods for the catalytic generation of reactive species using NHC-catalysts relies on internal, or intramolecular, redox reactions of functionalized starting materials. A similar concept can be applied to intermolecular redox reactions, in which two functional groups react selectively with one another in an exchange of oxidation states to give a new product. We believe that this concept, which combines functional groups that react selectively with one another, can form the basis of the discovery of new chemoselective ligation reactions (Scheme 11). Importantly, we consider the development and application of such reactions as the preeminent challenge in synthetic methodology as such methods are needed to expand the horizons of size and complexity that can currently be accessed by synthetic organic chemists.

The need for such reactions arises because classical, stepwise synthetic approaches to complex molecules are largely restricted to the practical preparation of relatively modest covalent assemblies. ${ }^{[25]}$ Larger structures (mw > 2000) must be prepared either through biotechnological approaches, which are constrained by biosynthetic pathways, or by polymerization strategies that are not amenable to precise control of the resulting products or incorporation of diverse functional groups. The lack of reliable methods to access defined organic structures of higher molecular weight and complexity presently represents the dividing line between what chemists are capable of creating and the intricate complexities necessary for such processes as molecular replication, photosynthesis, and chemical recognition. New chemical approaches that must meet a strict set of criteria are needed to make possible the controlled synthesis of structurally defined, highly functionalized systems that will blur the boundaries between the capabilities of synthetic and living systems.
The few reactions that meet or approach the strict criteria of chemical ligation, such as the $\mathrm{Cu}$ catalyzed coupling of azides and alkynes, have already had a profound effect on the synthesis of biologically active molecules and materials.

In our initial efforts in this area, we have succeeded in developing one of the first general methods for amide-forming chemical ligation under aqueous conditions without the use of reagents or the production of byproducts (Scheme 12). ${ }^{[26]}$ Our preliminary results demonstrate the potential of this process to significantly impact the preparation of proteins, glycopeptides, and related structures by the highly selective coupling of unprotected fragments. The utility of this ligation for complex molecule synthesis is further supported by numerous ongoing projects in our lab.

In order to make the application of this reaction to peptide and protein synthesis a reality, efficient and transparent methods for the preparation of the key precursors, $C$ terminal peptide $a$-ketoacids and N-terminal hydroxylamines, are needed. We found that existing approaches to these functional groups in the context of complex, unprotected peptides failed to provide reliable routes to these materials. To address this, we have engaged in a program of reaction discovery and development to fulfill these needs. These studies have resulted in a new approach to the synthesis of enantiomerically pure peptide $a$-ketoacids ${ }^{[27]}$ (Scheme 13 ) and $\mathrm{N}$-terminal hydroxylamines ${ }^{[28]}$ that interface with established, Fmoc-based solid phase peptide synthesis (Scheme 14).

We are also applying these innovations to new methods for the synthesis of peptidomimetics and biocompatible materials. For example, the ability to prepare polyamide and polyester based structures under mild, aqueous conditions and without the need for transition metal catalysts is currently an unmet challenge. In preliminary efforts towards this goal, we have developed a unique approach to the synthesis of peptide oligomers under aqueous conditions. ${ }^{[29]}$ Coupling of an $a$-ketoacid with 


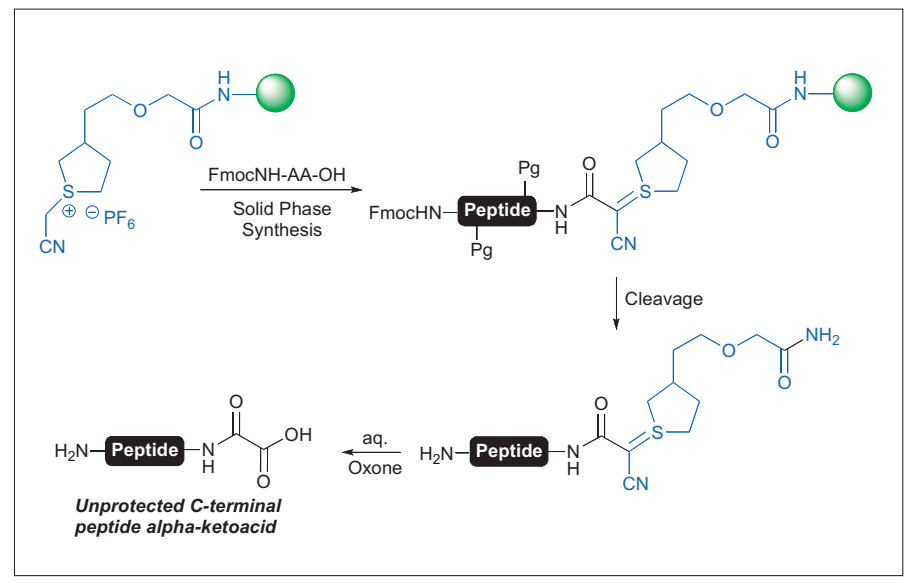

Scheme 13. Solidphase synthesis of C-terminal peptide $\alpha$-ketoacids.

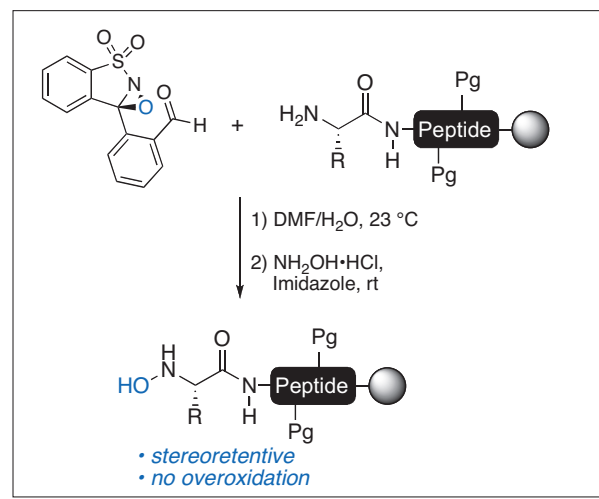

Scheme 14. A reagent for the solid-phase synthesis of peptide hydroxylamines. isoxazolidine monomers initiates a chain reaction that, upon peptide formation, generates a new $a$-ketoacid (Scheme 15). This reaction, which occurs in a wide range of solvents and $\mathrm{pH}$, has great potential for the synthesis of peptidomimetics and new biomaterials. We are presently developing solid supported variants for the iterative synthesis of longer peptides as well as pursuing new polymerization strategies. Importantly, our evidence to date suggests that we will be able to employ highly functionalized monomers that would normally not be amenable to oligomerization strategies with any other approach. In future work, we plan to greatly extend this reaction to include new monomer classes suitable for the synthesis of novel peptidic structures.

\section{Project 3: Design, Synthesis, and Applications of Adaptive Organic Molecules}

Dynamic chemical systems that adapt and evolve to generate novel characteristics are an emerging approach to the rapid discovery of molecules with customized properties. A major barrier of the implementation of such systems is the limited and relatively harsh conditions under which reversible covalent bond formation can occur. We reasoned that this ideal would most easily be achieved in a 'selfcontained' dynamic combinatorial library where distinct spatial arrangements of key functional groups or recognition elements would arise from spontaneous intramolecular rearrangement processes. In this regard, a polysubstituted bullvalene would provide a unique and robust dynamic platform in which hundreds or thousands of constitutional isomers could be accessed by facile valence tautomerization reactions (Fig. 3). We have recently developed a concise, scalable synthesis of a polysubstituted bullvalene precursor in which dynamic behavior can be modulated by external control.

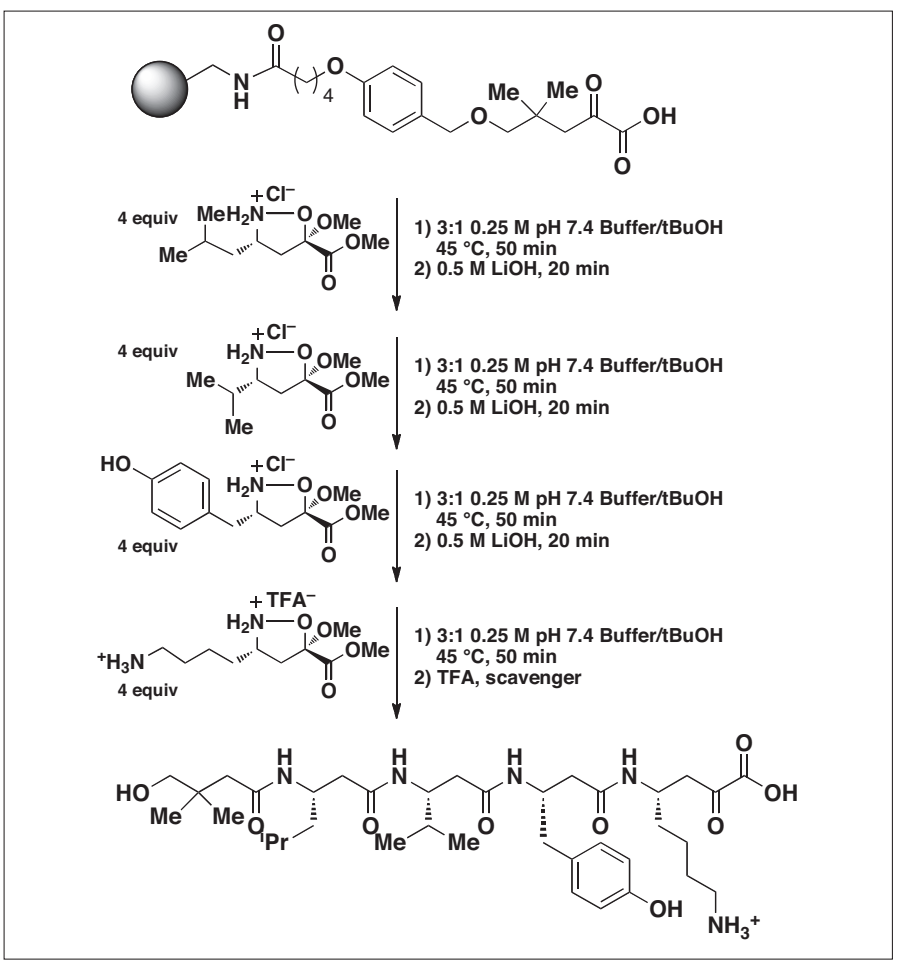

Scheme 15. Iterative, reagent-less synthesis of $\beta^{3}$-oligopptide.

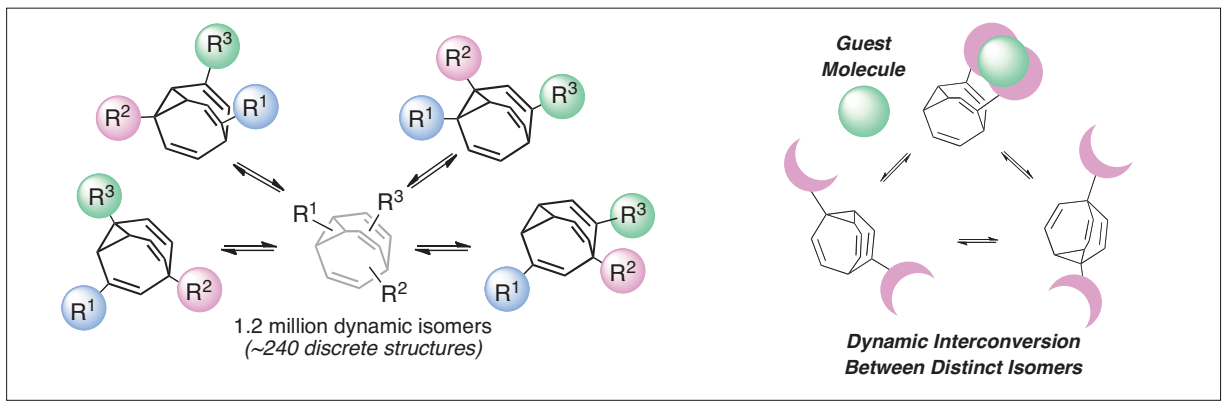

Fig. 3. (a) Dynamic interconversion of bullvalone isomers via base-generated hydroxybullvalenes. (b) Dynamic complexation of a guest molecule by shape-shifting host molecules.

Although such systems have been previously postulated, the synthetic challenge posed by preparing, modifying, and modulating a polysubstituted dynamic platform has thus far prevented the implementation and evaluation of this strategy. We approached the polysubstituted bullvalene problem from the viewpoint of complex molecule synthesis and developed a mod- ern, practical method for its preparation and late stage functionalization (Scheme 16). ${ }^{[30]}$ With a scalable and flexible method for the preparation of oligosubstituted bullvalenes in hand, we have begun to explore the application of these remarkable compounds. Our initial proof of principle studies on their ability to adapt to a substrate to give tightly binding compounds demon- 


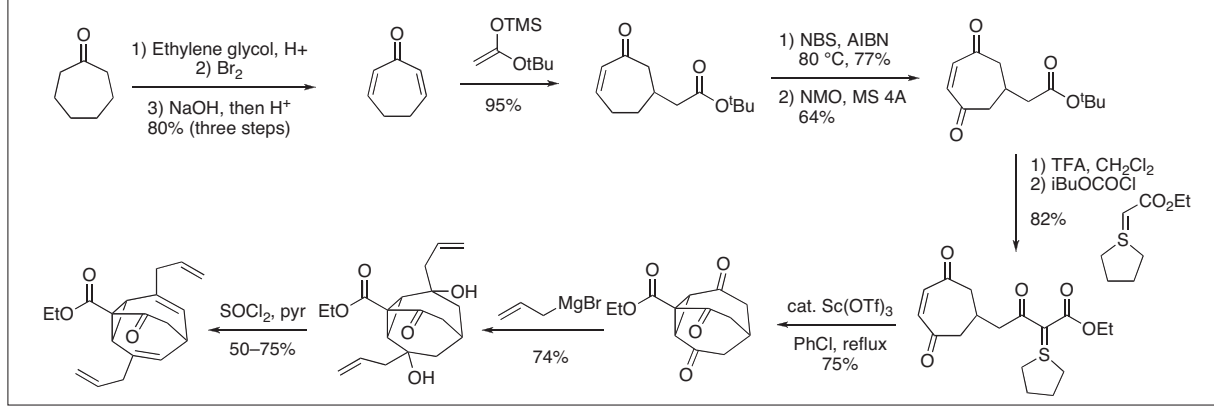

Scheme 16. Rational, scalable synthesis of a polysubstituted bullvalone.

strated that they may have potential as synthetic antibodies, although further studies on less biased systems are needed. More recently, we are pursuing their use as sensor and exploiting their ability to adapt to small changes in their local environment. Given the unprecedented properties of these molecules, we anticipate many years of discovery and application to come.

\section{Conclusions and Future Directions}

For much of its history, organic chemistry, particularly synthetic organic chemistry, has been thought of as difficult, unpredictable, unforgiving, not to mention messy, smelly, dangerous, and toxic. Certainly some of these aspects will always be true, but the remarkable progress of synthetic chemistry and the discovery of new reactions have shown that this does not have to be the status quo. Despite these successes, there is still a long way to go before synthetic chemists can assemble complex structures with the specificity, speed, and flexibility needed to rival biology's ability to evolve catalysts, materials, and pharmacologically active molecules. The role of reaction discovery must not be forgotten. We hope to continue enjoying the small successes towards better methods while at the same time realizing that these are only very small steps towards a larger challenge.

Received: February 12, 2011

[1] For example: a) B. List, Tetrahedron 2002, 58 , 5573; b) B. List, Acc. Chem. Res. 2004, 37 548; c) W. Notz, F. Tanaka, C. F. Barbas, Acc. Chem. Res. 2004, 37, 580; d) S. Bertelsen, K. A. Jorgensen, Chem. Soc. Rev. 2009, 38, 2178; e) B. M. Trost, C. S. Brindle, Chem. Soc. Rev. 2010, 39, 1600.

[2] a) D. E. Frantz, R. Fassler, C. S. Tomooka, E. M. Carreira, Acc. Chem. Res. 2000, 33, 373; b) H. Sasaki, D. Boyall, E. M. Carreira, Helv. Chim. Acta. 2001, 84, 964; c) R. Fassler, C. S. Tomooka, D. E. Frantz, E. M. Carreira, Proc. Natl. Acad. Sci. 2004, 101, 5843.

[3] a) H. Y. Jang, M. J. Krische, Acc. Chem. Res. 2004, 37, 653; b) M. Y. Ngai, J. R. Kong, M. J. Krische, J. Org. Chem. 2007, 72, 1063; c)
E. Skucas, M. Y Ngai, V. Komanduri, M. J. Krische, Acc. Chem. Res. 2007, 40, 1394; d) J. F. Bower, I. S. Kim, R. L. Patman, M. J. Krische, Angew. Chem. Int. Ed. 2009, 48, 34.

[4] For a review of catalyzed addition of nucleophiles to ketenes affords $\alpha$-chiral, enantiomerically enriched carboxylic acid derivatives, see R. K. Orr, M. A. Calter, Tetrahedron 2003, 59, 3345.

[5] The term activated carboxylate is taken to encompass any acyl species that reacts irreversibly with nucelophiles to give stable carboxylic acid derivates. Examples include acyl halides, acyl cyanides, and activated esters.

[6] For Lewis and Brønsted acid catalyzed esterifications and amidations of carboxylic acids, see: a) J. Otera, Angew. Chem. Int. Ed. 2001, 40, 2044; b) K. Ishihara, S. Ohara, H. Yamamoto, Science 2000, 290, 1140; URL: http://www. sciencemag.org/cgi/reprint/290/5494/1140; c) K. Wakasugi, T. Misaki, K. Yamada, Y. Tanabe, Tetrahedron Lett. 2000, 41, 5249; d) J. Otera, 'Esterification: Methods, Reactions, and Applications', Wiley \& Sons, New York, 2003.

[7] a) K. Daigo, L. J. Reed, J. Am. Chem. Soc. 1962, 84,659 ; b) T. C. Owen, A. Richards, J. Am. Chem. Soc. 1987, 109, 2520; b) T. C. Owen, J. N. Harris, J. Am. Chem. Soc. 1990, 112, 6136.

[8] a) M. Movassaghi, M. A. Schmidt, Org. Lett. 2005, 7, 2453; b) S. De Sarkar, S. Grimme, A. Studer, J. Am. Chem. Soc. 2010, 132, 1190.

[9] a) J. W. Bode, S. S. Sohn, J. Am. Chem. Soc. 2007, 129, 13798; b) H. U. Vora, T. Rovis, $J$. Am. Chem. Soc. 2007, 129, 13796

[10] P.-C. Chiang, Y. Kim, J. W. Bode, Chem. Commun. 2009, 4566.

[11] For example: a) B. M. Trost, H. Ito, J. Am. Chem. Soc. 2000, 122, 12003; b) N. Kumagai, S. Matsunaga, N. Yoshikawa, T. Ohshima, M. Shibasaki, Org. Lett. 2001, 3, 1539; c) B. M. Trost, D. W. Lupton, Org. Lett. 2007, 9, 2023; d) S. Matsunaga, M. Shibasaki, Bull. Chem. Soc. Jpn. 2008, 81, 60.

[12] S. Mukherjee, J. W. Yang, S. Hoffmann, B. List, Chem. Rev. 2007, 107, 5471.

[13] a) M. He, J. R. Struble, J. W. Bode, J. Am. Chem. Soc. 2006, 128, 8418; b) M. He, G. J. Uc, J. W. Bode, J. Am. Chem. Soc. 2006, 128, 15088; c) M. He, B. J. Beahm, J. W. Bode, Org. Lett. 2008, 10, 3817; d) J. Kaeobamrung, M. C. Kozlowski, J. W. Bode, Proc. Natl. Acad. Sci. 2010, 107, 20661.

[14] a) L. He, H. Lv, Y.-R. Zhang, S. Ye, J. Org. Chem. 2008, 73, 8101; b) N. Duguet, C. D. Campbell, A. M. Z. Slawin, A. D. Smith, Org. Biomol. Chem. 2008, 6, 1108; c) C. D. Campbell, N. Duguet, K. A. Gallagher, J. E. Thomson, A. G. Lindsay, A. C. O’Donoghue, A. D. Smith, Chem. Commun. 2008, 3528; d) Y.-R. Zhang, H. Lv, D. Zhou, S. Ye, Chem. Eur. J. 2008, 14, 8473; e) X.-N. Wang, P.-L. Shao, H. Lv, S. Ye, Org. Lett. 2009, 11, 4029.

[15] Y. Kawanaka, E. M. Phillips, K. A. Scheidt, J. Am. Chem. Soc. 2009, 131, 18028.
[16] a) S. S. Sohn, E. L. Rosen, J. W. Bode, J. Am. Chem. Soc. 2004, 126, 14370; b) C. Burstein, F Glorius, Angew. Chem. Int. Ed. 2004, 43, 6205; c) S. S. Sohn, J. W. Bode, Org. Lett. 2005, 7, 3873.

[17] For example from others: a) A. Chan, K. A. Scheidt, J. Am. Chem. Soc. 2007, 129, 5334; b) E. M. Phillips, T. E. Reynolds, K. A. Scheidt, J. Am. Chem. Soc. 2008, 130, 2416; c) V. Nair, B. P. Babu, S. Vellalath, E. Suresh, Chem. Commun. 2008, 747; d) V. Nair, C. R. Sinu, B. P. Babu, V. Varghese, A. Jose, E. Suresh, Org. Lett. 2009, 11, 5570 .

[18] a) P.-C. Chiang, J. W. Bode in 'N-Heterocyclic Carbenes: From Laboratory Curiosities to Efficient Synthetic Tools', Ed. S. DíezGonzález, Royal Society of Chemistry, Cambridge, 2010; b) J. L. Moore, T. Rovis in 'Topics in Current Chemistry, Vol. 291', Ed. B. List, Springer, Berlin, 2009; c) V. Nair, S. Vellalath, B. P. Babu, Chem. Soc. Rev. 2008, 37, 2691.

[19] M. He, J. W. Bode, Org. Lett. 2005, 7, 3131.

[20] a) M. Rommel, T. Fukuzumi, J. W. Bode, J. Am. Chem. Soc. 2008, 130, 17266; b) P. Zheng, C. A. Gondo, J. W. Bode, Chem. Asian J. 2011, 6, 614.

[21] a) P.-C. Chiang, J. Kaeobamrung, J. W. Bode, J. Am. Chem. Soc. 2007, 129, 3520; b) M. He, J. W. Bode, J. Am. Chem. Soc. 2008, 130, 418; c) J. Kaeobamrung, J. W. Bode, Org. Lett. 2009, 11, 677.

[22] For an alternative interpretation of the mechanism, see V. Nair, S. Vellalath, M. Poonoth, E. Suresh, J. Am. Chem. Soc. 2006, 128, 8736.

[23] J. Mahatthananchai, P. Zheng, J. W. Bode, Angew. Chem. Int. Ed. 2011, DOI: 10.1002/ anie. 201005352.

[24] J. Kaeobamrung, J. Mahatthananchai, P. Zheng, J. W. Bode, J. Am. Chem. Soc. 2010, 132, 8810.

[25] C. J. Hawker, K. L. Wooley, Science 2005, 309, 1200.

[26] J. W. Bode, R. M. Fox, K. D. Baucom, Angew. Chem. Int. Ed. 2006, 46, 1248.

[27] a) L. Ju, A. R. Lippert, J. W. Bode, J. Am. Chem. Soc. 2008, 130, 4253; b) L. Ju, J. W. Bode, Org. Biomol. Chem. 2009, 7, 2259.

[28] T. Fukuzumi, J. W. Bode, J. Am. Chem. Soc. 2009, 131, 3864 .

[29] N. Carrillo, E. A. Davalos, J. A. Russak, J. W. Bode, J. Am. Chem. Soc. 2006, 128, 1452.

[30] A. R. Lippert, J. Kaeobamrung, J. W. Bode, J. Am. Chem. Soc. 2006, 128, 14738. 